Ter ahert $z$ char act er i zat $i$ on of sem conduct or al I oy Al I nN: negat i ve i magi nary conduct i vi ty and i ts meani ng

\begin{tabular}{|l|l|}
\hline 著者 & $\begin{array}{l}\text { KANG Ti ng- Ti ng, YAMANOTO Nasat ond, TANAKA } \\
\text { M ki yasu, HASH MTO Aki hi ro, Yamanot o Aki o, } \\
\text { SUTO Ryot a, NODA Aki f um, LI U D. W, YAMANOTO } \\
\text { Kohj i }\end{array}$ \\
\hline $\begin{array}{l}\text { j our nal or } \\
\text { publ i cat i on ti t l e }\end{array}$ & OPTI CS LETTERS \\
\hline vol une & 34 \\
\hline number & 16 \\
\hline page r ange & $2507-2509$ \\
\hline year & $2009-08$ \\
\hline URL & ht t p: //hdl . handl e. net $/ 10098 / 2124$ \\
\hline
\end{tabular}




\title{
Terahertz characterization of semiconductor alloy AlInN: negative imaginary conductivity and its meaning
}

\author{
Ting-Ting Kang, ${ }^{1, *}$ Masatomo Yamamoto, ${ }^{1}$ Mikiyasu Tanaka, ${ }^{1}$ Akihiro Hashimoto, ${ }^{1}$ Akio \\ Yamamoto, ${ }^{1}$ Ryota Suto, ${ }^{2}$ Akifumi Noda, ${ }^{2}$ D. W. Liu ${ }^{2}$ and Kohji Yamamoto ${ }^{2}$ \\ ${ }^{I}$ Department of Electrical and Electronics Engineering, Graduate School of Engineering, \\ University of Fukui, Bunkyo 3-9-1, Fukui 910-8507, Japan \\ ${ }^{2}$ Research Center for Development of Far-Infrared Region, University of Fukui, Fukui, Japan \\ *Corresponding author: ktt219@163.com
}

\begin{abstract}
:
Through terahertz time-domain spectroscopy, negative imaginary conductivity is observed in In-rich AlInN film grown by metal-organic chemical vapor deposition for frequencies from 0.2 to $2.0 \mathrm{THz}$. This non-Drude behavior is explained based on the electron back scattering theory of N. V. Smith. Comparing with binary semiconductor InN, potential fluctuations produced by composition inhomogeneity and alloy scattering of carriers make In-rich AlInN alloy easier subjected to non-Drude behavior in electrical performance.
\end{abstract}

OCIS codes: $300.6470,300.6495,310.6860$ 
Using noncontact terahertz time-domain spectroscopy (THz-TDS) to study the carrier dynamics of semiconductor is an important field. There have been a lot of reports on terahertz (THz) spectroscopy of binary semiconductors, e.g. $\mathrm{ZnO}[1], \mathrm{GaN}[2,3]$, or nearly binary semiconductors with very low alloy composition, e.g. GaNAsBi with $\sim 1 \% \mathrm{~N}, \mathrm{Bi}[4]$. The present paper will give some knowledge of ternary semiconductor AlInN alloy with relative high alloy composition $\sim 20 \%$ in $\mathrm{THz}$ spectroscopy.

There have been some reports on the applications of $\mathrm{InN}$ and its In-rich alloys in $\mathrm{THz}[5,6]$ and high-frequency electronics[7]. For semiconductors, ultrafast photon generated electron acceleration is a general way to produce $\mathrm{THz}$ emission, which leading to the dependence of THz-radiation power $P$ on effective electron mass $m^{*}$ as: $P \propto\left(\frac{1}{m^{*}}\right)^{2}$ [8]. On the other hand, the narrow bandgap of In-rich nitride will facilitate the use of 1.3-1.5 $\mu \mathrm{m}$ laser as $\mathrm{THz}$ excitation sources, and integrate $\mathrm{THz}$ technology with fiber-based telecommunication system. Therefore, considering the small effective mass $m^{*}=0.075 m_{0}\left(m_{0}\right.$ is the free electron mass) [9] and narrow bandgap $0.7 \mathrm{eV}$ of $\mathrm{InN}$, In-rich nitride is regarded as an attractive candidate for high power $\mathrm{THz}$ sources.

Here, using THz-TDS, we study the optical properties of In-rich AlInN in THz frequency and extract related electrical information. In order to simplify the problem involving multi-layer THz wave reflection and absorption, we don't use any conductive buffer or template, e. g. widely used GaN buffer/template, to grow AlInN. After substrate thermal cleaning and nitridation, AlInN films were directly grown by metal-organic chemical vapor deposition (MOCVD) on Cplane sapphire, with pressure around 730 torr and growth temperature around $600^{\circ} \mathrm{C}$. All $\mathrm{THz}$ measurements are conducted in room temperature. The THz electromagnetic wave are produced 
and detected by photoconductive antennas fabricated on low-temperature-grown GaAs films. For reference signal, $\mathrm{THz}$ pulses transmitted through the sapphire substrate without growth is measured.

For the X-ray diffraction (XRD) spectra shown in Fig. 1(a), since no buffer or interlayer was used, only the signals from the AlInN film and sapphire substrate are observed. The Alcontent $\mathrm{x}=0.2$ is obtained through Vegard's law. Fig. 1(b) plots the measured THz pulses transmitted through the AlInN sample and the sapphire substrate. In Fig. 1(c), the THz pulse spectrum in frequency domain is obtained by applying a fast Fourier transform (FFT) to the time-domain waveform.

In the simplest configuration of only one layer AlInN with thickness $d$ on sapphire, the refraction index of AlInN $n_{2}+\mathrm{i} k_{2}$ is determined from the following equation:

$$
\begin{aligned}
& \frac{E_{\text {film }}(\omega)}{E_{\text {ref. }}(\omega)}=\frac{\frac{t_{12} t_{23} \exp \left[i\left(n_{2}+i k_{2}\right) \omega d / c\right]}{1-r_{21} r_{23}\left[i 2\left(n_{2}+i k_{2}\right) \omega d / c\right]}}{t_{13} \exp \left(i n_{1} \omega d / c\right)} \\
& \approx 1+\frac{i \omega d}{c}\left[\frac{\left(n_{2}+i k_{2}\right)^{2}-n_{1}^{2}}{n_{1}+n_{3}}\right]
\end{aligned}
$$

where 1, 2, 3 denote the medium of air, nitride film, sapphire substrate respectively; $r_{i j}$ and $t_{i j}$ are the reflection and transmission coefficients of the $i \rightarrow j$ interface $(i, j=1,2$, and 3 , and $i \neq j)$, respectively; $E_{f i l m}(\omega)$ is the signal (with the film and substrate) field; $E_{\text {ref. }}(\omega)$ is the reference field (substrate only) ; $n_{1}=1, n_{3}=3.2$ are the refraction index of air and sapphire substrate respectively; $\omega$ is the radial frequency. During deducing Eq.(1), the approximation AlInN thickness $d=250 \mathrm{~nm}<<\lambda / n_{2}(\lambda \sim 300 \mu \mathrm{m}$ is the incident THz wavelength in air $)$ is used. The thickness of sapphire is $400 \mu \mathrm{m}$. A reference for the derivation of Eq.(1) and the THz-TDS system can be seen in Ref.(2). 
The complex dielectric constant $\varepsilon(\omega)$ is defined as:

$$
\varepsilon(\omega)=\left(n_{2}+\mathrm{i} k_{2}\right)^{2}=\varepsilon_{\infty}+i \frac{\sigma}{\omega \varepsilon_{0}}
$$

where $\varepsilon_{0}$ is the permittivity of free space, $\sigma$ is the complex conductivity. Since AlInN here only has $\mathrm{Al}$ content $\mathrm{x}=0.2$, the dielectric constant $\varepsilon_{\infty}=6.7$ for $\operatorname{InN}[9]$ is adopted. The results below will be revised a little if $\varepsilon_{\infty}=4.16$ for AlN [10] is used.

Using the analysis method above to treat the original data, the complex conductivity of $\mathrm{Al}_{0.2} \mathrm{In}_{0.8} \mathrm{~N}$ is presented in Fig. 1(d). As shown, the original data is subject to oscillation due to multiple reflections. In order to remove this useless oscillation structures, we will make a cut in time domain. Since the main THz pulse is well separated from reflected pulse in Fig.1(b), the extraction was done with the data values recorded only up to $\sim 16 \mathrm{ps}$. This time-domain cut procedure leads to a satisfactorily remove of oscillation structures, which can be seen in Fig.1 (c). At the same time, the $\omega / 2 \pi$ frequency resolution retreats from $\sim 0.015 \mathrm{THz}$ to $\sim 0.06 \mathrm{THz}$.

The refractive index and complex conductivity of AlInN after time-domain cut are presented in Fig.2 (a, b). For comparison, InN with 800nm thickness prepared under similar growth conditions, except a thin insulative AlN buffer (thickness $\sim 10 \mathrm{~nm}$ ) to improve morphology, is measured by THz-TDS and its results are also given in Fig.2. We can see that the imaginary part of complex conductivity $(\operatorname{Im}[\sigma])$ of $A l \operatorname{InN}$ is negative with the approximate relation $\operatorname{Im}[\sigma]=-a \omega(a>0)$. While $\operatorname{Im}[\sigma]$ of $\operatorname{InN}$ is always around zero, nearly independent of frequency $\omega / 2 \pi$. It should be noted that the negative $\operatorname{Im}[\sigma]$ in AlInN has nothing to do with the time-domain cut procedure, because $\operatorname{Im}[\sigma]$ without time-domain cut in Fig.1(d) also display negative values in high frequency. 
The negative $\operatorname{Im}[\sigma]$ can not be explained by simple Drude model $\sigma(\omega)=\varepsilon_{0} \omega_{p}^{2} \tau_{0} /\left(1-i \omega \tau_{0}\right)$, where $\omega_{p}$ is the plasma frequency; $\tau_{0}$ is the velocity relaxation time. This job can be done by Drude-Smith model [11] - a relatively simple classical model describing systems in which negative $\operatorname{Im}[\sigma]$ is accommodated by incorporating memory effects in the scattering process. As a usual practice, it assumes that persistence of velocity is retained for only one collision. Therefore, the complex conductivity in the Drude-Smith formalism is given by:

$$
\sigma(\omega)=\frac{\varepsilon_{0} \omega_{p}^{2} \tau_{0}}{\left(1-i \omega \tau_{0}\right)}\left(1+\frac{C}{1-i \omega \tau_{0}}\right)=\frac{\varepsilon_{0} \omega_{p}^{2} \tau_{0}}{\left(1+\omega^{2} \tau_{0}^{2}\right)^{2}}\left[(1+C)+(1-C) \omega^{2} \tau_{0}^{2}+i \omega \tau_{0}\left(1+2 C+\omega^{2} \tau_{0}^{2}\right)\right]
$$

where $C$ is a parameter ranged from -1 (back scattering only) to 0 (Drude scattering only), describing the persistence of velocity after the only scattering event. Previously, Drude-Smith model has been used to interpret negative $\mathrm{THz} \operatorname{Im}[\sigma]$ in nanostructures $[12,13,14]$, where the nanometer scale size directly provides carrier localization. In such cases, $C$ value can even reach -0.98 . Therefore, in other words, $C$ can be regarded as a parameter characterizing the extent of carrier localization.

When $\omega \tau_{0}<<1$, Eq.(3) can be simplified further into:

$$
\sigma(\omega)=\varepsilon_{0} \omega_{p}^{2} \tau_{0}\left[(1+C)+i \omega \tau_{0}(1+2 C)\right]
$$

Eq. (4) is account for the fact that $\operatorname{Im}[\sigma]=-a \omega$ for AlInN. In Fig.2(b), $\sigma_{\text {AlInN }}$ is fitted with $\omega_{p} / 2 \pi=240 \mathrm{THz}, C=-0.82, \tau_{0}=8.0$ fs. Comparing with the fitting parameters of $\operatorname{InN}\left(\omega_{p}\right.$ $\left./ 2 \pi=90 \mathrm{THz}, C=-0.45, \tau_{0}=13.5 \mathrm{fs}\right), \mathrm{Al}_{0.2} \mathrm{In}_{0.8} \mathrm{~N}$ demonstrates higher electron concentration, lower mobility and stronger back scattering. In the case of only Drude-like scattering $C=0$, using Eq.(3), the static Re $\sigma(0)$ can be enhanced considerately from $\sim 300$ to $\sim 1600 \Omega^{-1} \mathrm{~cm}^{-1}$ for AlInN, which indicates that back scattering is an important scattering mechanism in semiconductor alloy. 
From Eq.(4), $C \sim-0.5$ is proposed to be responsible for the nearly frequency independence of $\operatorname{Im}[\sigma] \sim 0$ in our $\operatorname{InN}$ sample. The more negative $C$ value than $\mathrm{InN}$ indicates stronger localization of charge carriers in AlInN.

Comparing Fig.2(b) with Ahn's work on InN film [15], where $\tau_{0}=52$ fs, the relaxation time $\tau_{0}=13.5$ fs and the derived mobility is both small for our InN samples. This low mobility, in combination with relative larger backscattering, greatly reduce the $\operatorname{Re}[\sigma], \operatorname{Im}[\sigma]$ and the resultant $\mathrm{THz}$ absorption characterized by $k$ - imaginary part of refractive index. Therefore, although $n$ (the real part of refractive index) of our sample is nearly same as Ahn's work, but $k$ value is smaller than their results. The inferior quality of present InN sample is resulted from the factor that we only adopt very thin $\sim 10 \mathrm{~nm}$ AlN buffer or no buffer for InN, InAlN MOCVD growth. If we use AlN or GaN template, the sample quality can be improved significantly. But in those cases, the THz response of the film will be mixed with those signals from the templates, which adding some complexity and uncertainty to the mathematical extraction procedure.

The most interesting consequence of negative $\operatorname{Im}[\sigma]$ is the reverse direction of current $j(t)$ before relaxing to its equilibrium value of zero. Let us imagine a unit impulse of electric field applied to the electron system at time $t=0$, the resulting current response $j(t)$ and complex conductivity $\sigma(\omega)$ are Fourier transforms of each other: $j(t) \propto \int_{0}^{\infty} \widetilde{\sigma}(\omega) e^{-i \omega t} d t$. From Eq.(3), as shown in Ref.(11),we have $j(t) / j(0)=\left(1+C \frac{t}{\tau_{0}}\right) e^{-t / \tau_{0}}$, which predicts that the present AlInN film will display maximum reverse current around $15 \mathrm{fs}$ after the beginning of current impulse. 
However, the observation of this reverse current depends critically on how well $\sigma(\omega)$ is described by Eq.(3) in higher frequency range. For example $\operatorname{Im}[\sigma]$ should rise to positive values when $1+2 C+\omega^{2} \tau_{0}^{2}>0$ is satisfied. Here it is $\omega / 2 \pi>16 \mathrm{THz}$ for AlInN. Unfortunately, this value is well beyond the frequency coverage of our THz-TDS setup and it cannot give reliable results in such high-frequency range.

In nanostructures, the carrier confinement by $\mathrm{nm}$ scale structure size provides a natural explanation to the negative $\operatorname{Im}[\sigma]$ in $\mathrm{THz}$. For semiconductor alloy, potential fluctuations produced by composition inhomogeneity will also result in such carrier localization. Alloy scattering, which is present even in a perfect alloy, is another mechanism to enhance this localization. Although for the high electron concentration $\mathrm{n} \sim 10^{19-20} \mathrm{~cm}^{-3}$ in our AlInN sample, alloy scattering may lose its dominance to Coulomb scattering [16]. It can still make significant contribution to reduce electron mobility $\mu$. Correspondingly, diffusion coefficient $D=\frac{k_{B} T}{e} \mu$ is suppressed, which contributes to carrier localization in another way.

High-frequency measurements can probe conductivity over shorter length scales given by $L_{\omega}=\sqrt{D / \omega}$ [17]. Accordingly, in higher frequency, short-range transport, which is less sensitive to potential fluctuations, plays more important roles in the overall transport than longrange transport. And the influence of potential fluctuations will be weakened under an increasing $\omega$. For AlInN, in Fig. 2(b), a weak but obvious enhancement of $\operatorname{Re}[\sigma]$ with increased $\omega$ is observed, evidencing the existence of carrier localization. Because such potential fluctuations are absent in InN, and in combination of the situation $\omega \tau_{0}<<1$ for the $\operatorname{InN}$ sample, $\operatorname{InN} \operatorname{Re}[\sigma]$ is almost constant in $0.2-2 \mathrm{THz}$. This frequency independent $\mathrm{Re}[\sigma]$ has ever been observed in $\mathrm{ZnO}$ 
film[1], where the authors proposed a broadband terahertz pulse reflector based on this frequency independence.

In conclusion, the optical constants and conductivity of $\mathrm{AlInN}$ in $\mathrm{THz}$ frequency are probed by THz-TDS. Negative imaginary conductivity is observed and explained based on electron back scattering theory of N. V. Smith. Potential fluctuations produced by composition inhomogeneity and alloy scattering are believed to enhance carrier localization in alloy, making non-Drude behavior easier to happen in semiconductor alloy.

This work was supported by the Ministry of Education, Culture, Sports, Science and Technology of Japan through Grant-in-Aid for Scientific Research in Priority Areas (No. 18069005).

\section{Reference:}

1. Guohong Ma, Dong Li, Hong Ma, Jie Shen, Chenguo Wu, Jin Ge, Shuhong Hu, and Ning Dai, "Carrier concentration dependence of terahertz transmission on conducting $\mathrm{ZnO}$ films," Appl. Phys. Lett. 93,211101 (2008).

2. Tsong-Ru Tsai, Shi-Jie Chen, Chih-Fu Chang, Sheng-Hsien Hsu, Tai-Yuan Lin, ChengChung Chi, "Terahertz response of GaN thin films," Opt. Express 14, 4898 (2006).

3. W. Zhang, A. K. Azad, and D. Grischkowsky, "Terahertz studies of carrier dynamics and dielectric response of n-type, freestanding epitaxial GaN,” Appl. Phys. Lett. 82, 2841-2843 (2003). 
4. D. G. Cooke, F. A. Hegmann, E. C. Young, and T. Tiedje, "Electron mobility in dilute GaAs bismide and nitride alloys measured by time-resolved terahertz spectroscopy," Appl. Phys. Lett. 89, 122103 (2006).

5. Ricardo Ascázubi, Ingrid Wilke, Kyle Denniston, Hai Lu, and William J. Schaff, "Terahertz emission by InN,” Appl. Phys. Lett. 84, 4810 (2004).

6. H. Ahn, Y.-P. Ku, C.-H. Chuang, C.-L. Pan, H.-W. Lin, Y.-L. Hong, and S. Gwo, "Intense terahertz emission from a-plane InN surface,” Appl. Phys. Lett. 92, 102103 (2008).

7. Yu-Syuan Lin, Shun-Hau Koa, Chih-Yuan Chan, Shawn S. H. Hsu, Hong-Mao Lee, and Shangjr Gwo, "High current density InN/AIN heterojunction field-effect transistor with a SiNx gate dielectric layer,” Appl. Phys. Lett. 90, 142111 (2007).

8. Kiyomi Sakai(Ed.), Terahertz Optoelectronics (Springer-Verlag Berlin Heidelberg, 2005), Page 102.

9. A. Kasic, M. Schubert, Y. Satio, Y. Nanishi, and G. Wagner, "Effective electron mass and phonon modes in n-type hexagonal InN,” Phys. Rev. B 65, 115206 (2002).

10. W. J. Moore, J. A. Freitas, Jr., R. T. Holm, O. Kovalenkov, and V. Dmitriev, "Infrared dielectric function of wurtzite aluminum nitride,” Appl. Phys. Lett. 86, 141912 (2005).

11. N. V. Smith, "Classical generalization of the Drude formula for the optical conductivity," Phys. Rev. B 64, 155106 (2001).

12. D. G. Cooke, A. N. MacDonald, A. Hryciw, J. Wang, Q. Li, A. Meldrum, and F. A. Hegmann, "Transient terahertz conductivity in photoexcited silicon nanocrystal films," Phys. Rev. B 73, 193311 (2006).

13. M. Beard, G. M. Turner, J. E. Murphy, O. I. Micic, M. C. Hanna, A. J. Nozik, and C. A. Schmuttenmaer, "Electronic Coupling in InP Nanoparticle Arrays," Nano Lett. 3, 1695(2003). 
14. G. M. Turner, M. C. Beard, and C. A. Schmuttenmaer, "Carrier Localization and Cooling in Dye-Sensitized Nanocrystalline Titanium Dioxide, ” J. Phys. Chem. B 106, 11716 (2002).

15. H. Ahn, Y.-P. Ku, Y.-C. Wang, and C.-H. Chuang, S. Gwo, and Ci-Ling Pan, "Terahertz spectroscopic study of vertically aligned InN nanorods, ” Appl. Phys. Lett. 91, 163105 (2007). And the Erratum: Appl. Phys. Lett. 94, 189901 (2009).

16. L. Hsu, R. E. Jones, S. X. Li, K. M. Yu and W. Walukiewicz, "Electron mobility in InN and III-N alloys,” J. Appl. Phys. 102, 073705 (2007).

17. P. F. Henning, C. C. Homes, S. Maslov, G. L. Carr, D. N. Basov, B. Nikolić, and M. Strongin, "Infrared Studies of the Onset of Conductivity in Ultrathin Pb Films," Phys. Rev. Lett. 83, 4880 (1999).

\section{Figure caption}

Fig.1: (a) XRD $\theta / 2 \theta$ scan spectra of AlInN film. Measured THz pulse passing through AlInN film and reference sapphire substrate: (b) in time domain; (c) in frequency domain. (d) Complex conductivity of AlInN fims deduced from the original results in Fig. 1(b) without 16 ps cut in time domain.

Fig.2. Real and imaginary parts of: (a) refractive index and (b) conductivity of the InN, AlInN film. In Fig.2 (b), for AlInN, dashed lines correspond to the calculated results based on the Drude-Smith model Eq.(3) and the fitting parameters are: $\omega_{p} / 2 \pi=240 \mathrm{THz}, C=-0.82$, $\tau_{0}=8.0 \mathrm{fs}$. 
Figures

Fig. 1
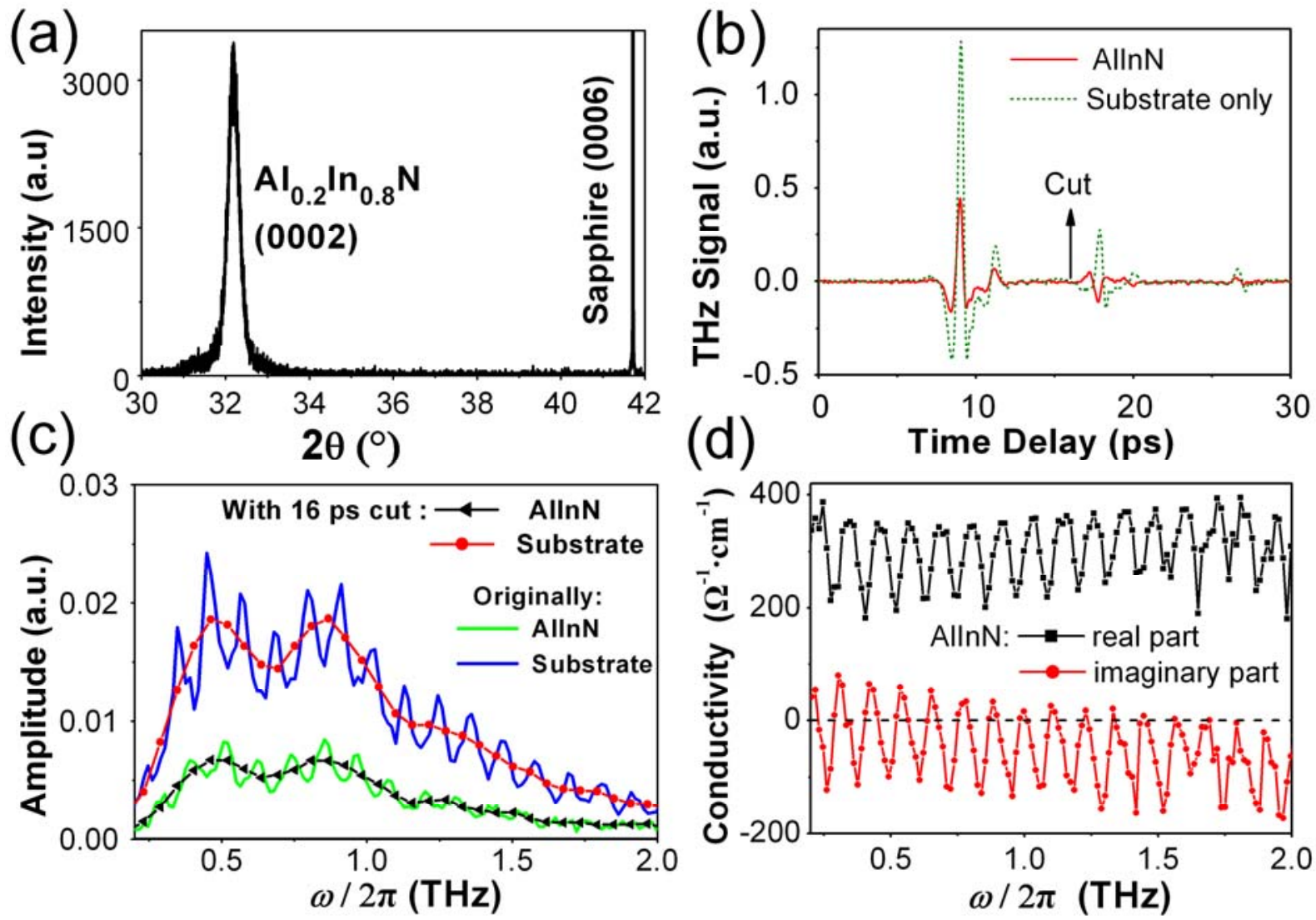

Fig. 2
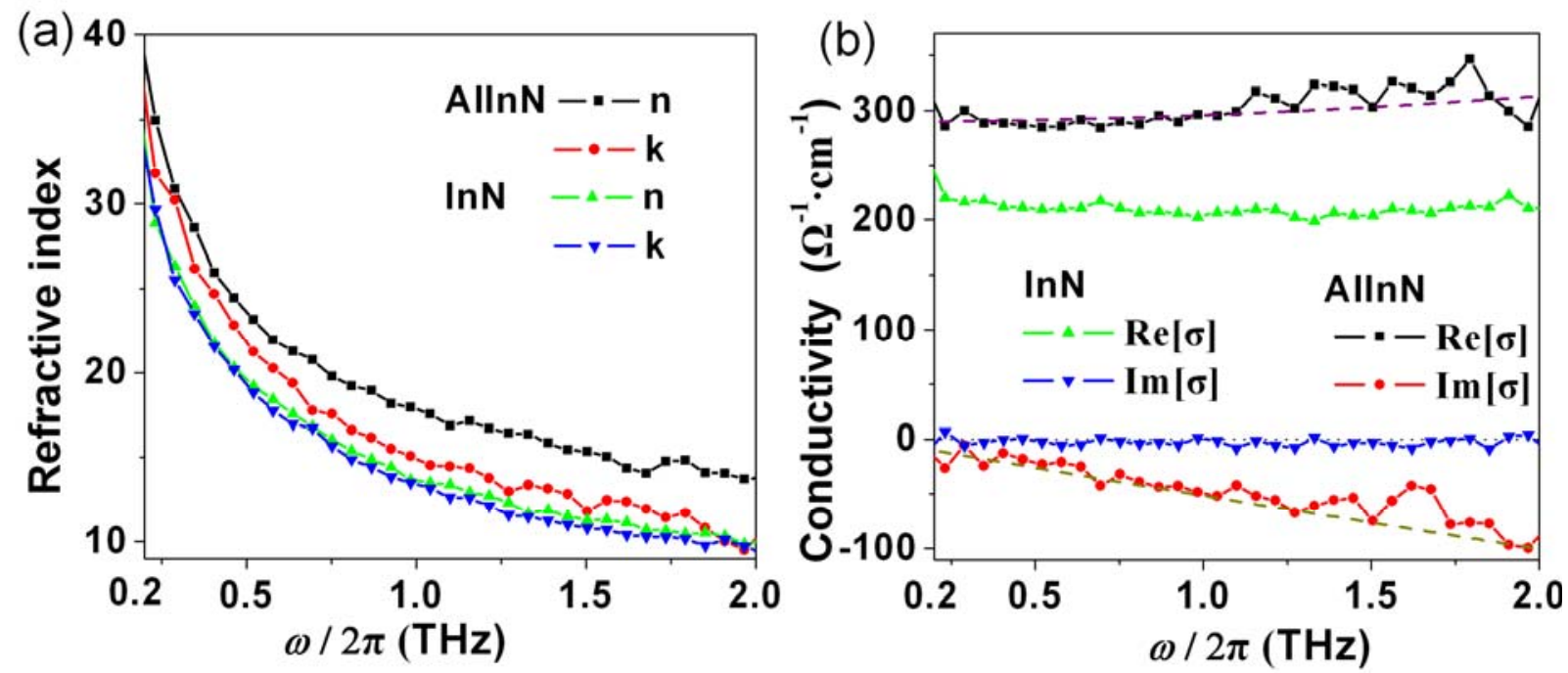